\title{
A NEW POLYNOMIAL INVARIANT OF KNOTS AND LINKS ${ }^{1}$
}

\author{
BY P. FREYD, D. YETTER; J. HOSTE; \\ W. B. R. LICKORISH, K. MILLETT; AND A. OCNEANU
}

The purpose of this note is to announce a new isotopy invariant of oriented links of tamely embedded circles in 3-space.

We represent links by plane projections, using the customary conventions that the image of the link is a union of transversely intersecting immersed curves, each provided with an orientation, and undercrossings are indicated by broken lines. Following Conway [6], we use the symbols $L_{+}, L_{0}, L_{-}$to denote links having plane projections which agree except in a small disk, and inside that disk are represented by the pictures of Figure 1.

Conway showed that the one-variable Alexander polynomials of $L_{+}, L_{0}$, $L_{-}$(when suitably normalized) satisfy the relation

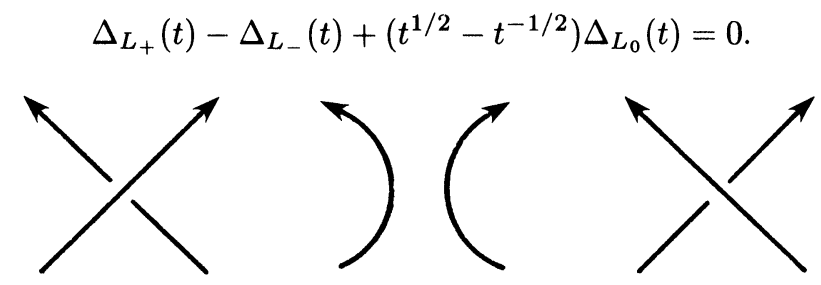

FIGURE 1

Received by the editors January 14, 1985.

1980 Mathematics Subject Classification. Primary 57M25.

${ }^{1}$ Editor's Note. The editors received, virtually within a period of a few days in late September and early October 1984, four research announcements, each describing the same result - the existence and properties of a new polynomial invariant for knots and links. There was variation in the approaches taken by the four groups and variation in corollaries and elaboratioul. These were: $A$ new invariant for knots and links by Peter Freyd and David Yetter; A polymomial invariant of knots and links by Jim Hoste; Topological invariants of knots and links, by W. B. R. Lickorish and Kenneth C. Millett, and A polymomial invariant for knots: $A$ combinatorial and an algebraic approach, by A. Ocneanu.

It was evident from the circumstances that the four groups arrived at their results completely independently of each other, although all were inspired by the work of Jones (cf. [10], and also [8, 9]). The degree of simultaneity was such that, by common consent, it was unproductive to try to assess priority. Indeed it would seem that there is enough credit for all to share in.

Each of these papers was refereed, and we would have happily published any one of them, had it been the only one under consideration. Because the alternatives of publication of all four or of none were both unsatisfying, all have agreed to the compromise embodied here of a paper carrying all six names as coauthors, consisting of an introductory section describing the basics written by a disinterested party, and followed by four sections, one written by each of the four groups, briefly describing the highlights of their own approach and elaboration. 
Recently, Vaughan Jones [10] constructed a new polynomial invariant satisfying the relation

$$
t V_{L_{+}}(t)-t^{-1} V_{L_{-}}(t)+\left(t^{1 / 2}-t^{-1 / 2}\right) V_{L_{0}}(t)=0
$$

Our invariant, which can be regarded either as a nonhomogeneous polynomial in two variables or a homogeneous polynomial in three variables, generalizes both the Alexander-Conway $[\mathbf{2 , 6}$ ] and the Jones polynomials.

MAIN THEOREM. There is a unique function $P$ from the set of isotopy classes of tame oriented links to the set of homogeneous Laurent polynomials of degree 0 in $x, y, z$ such that

(1) $x P_{L_{+}}(x, y, z)+y P_{L_{-}}(x, y, z)+z P_{L_{0}}(x, y, z)=0$,

(2) $P_{L}(x, y, z)=1$ if $L$ consists of a single unknotted component.

REMARKS. (1) If $L$ consists of $n$ unlinked and unknotted components, then

$$
P_{L}(x, y, z)=(-(x+y) / z)^{n-1} .
$$

(2) The Alexander-Conway polynomial of $L$ is

$$
\Delta_{L}(t)=P_{L}\left(1,-1, t^{1 / 2}-t^{-1 / 2}\right)
$$

and the Jones polynomial is

$$
V_{L}(t)=P_{L}\left(t,-t^{-1}, t^{1 / 2}-t^{-1 / 2}\right)
$$

(3) Since $P_{L}(x, y, z)$ is homogeneous, it can be viewed (in many ways) as a polynomial in two nonhomogeneous variables. A convenient way to do this is to set $P_{L}(l, m)=P_{L}\left(l, l^{-1}, m\right)$. In this notation the basic relation is $l P_{L_{+}}(l, m)+l^{-1} \mathcal{P}_{L_{-}}(l, m)+m \mathcal{P}_{L_{0}}(l, m)=0$.

(4) Reversing the orientation of $\mathbf{R}^{3}$ has the effect

$$
P_{L}(x, y, z) \leftrightarrow P_{L}(y, x, z), \quad \rho_{L}(l, m) \leftrightarrow \rho_{L}\left(l^{-1}, m\right) .
$$

However, neither polynomial is changed by simultaneous reversal of orientations on all components of $L$.

(5) If $L$ is a connected sum of links $L_{1}$ and $L_{2}$, then $P_{L}=P_{L_{1}} P_{L_{2}}$.

(6) The following examples are easily computed by using the recurrence relation and the values of $P$ for unlinks.

$$
\begin{aligned}
& L=\square: P_{L}=y z^{-1}+x^{-1} y^{2} z^{-1}-x^{-1} z \\
& L=P_{L}=x^{-2} z^{2}-2 x^{-1} y-x^{-2} y^{2} \\
& L=: P_{L}=y^{-2} z^{2}-2 x y^{-1}-x^{2} y^{-2} \\
& L=P_{L}=x^{-1} y^{-1} z^{2}-x y^{-1}-x^{-1} y-1 .
\end{aligned}
$$


We wish to express our appreciation to Vaughan Jones for communicating his results to us. The particular viewpoints of the various authors are outlined below.

\section{THE FREYD-YETTER APPROACH}

In order to reduce the unique existence of the homfly invariant to a word problem, let $R$ be the ring of Laurent polynomials on $x, y, z ; W_{n}$ the set of words on $n-1, \ldots, 2,1,-1, \ldots,-n+1$ (no zero); $W$ the disjoint union of the $W_{n}$ 's; $F$ the free $R$-module generated by $W$. An element of $W$ is denoted $[u]_{n}$, where $u \in W_{n} . R \rightarrow F$ denotes the map that sends 1 to [ $]_{1} . F \rightarrow M$ denotes the quotient module obtained by imposing the following relations, in which $u, v \in W_{n}$ and $0<b<a<n$ :

$$
\begin{aligned}
& {[u a(-a) v]_{n} \equiv[u v]_{n} \equiv[u(-a) a v]_{n}} \\
& {[u a b v]_{n} \equiv[u b a v]_{n} \text { if } b<a-1,} \\
& {[u a(a-1) a v]_{n} \equiv[u(a-1) a(a-1) v]_{n}} \\
& {[u v]_{n} \equiv[v u]_{n}} \\
& {[u n v]_{n+1} \equiv[u v]_{n} \equiv[u(-n) v]_{n+1}} \\
& x[u a v]_{n}+y[u(-a) v]_{n}+z[u v]_{n} \equiv 0 .
\end{aligned}
$$

LEMMA. $R \rightarrow F \rightarrow M$ is an isomorphism.

This lemma implies the Main Theorem by the following argument. Each element of $W_{n}$ describes a braid on $n$ strands. By the classic theorem of Artin $[\mathbf{1}]$, the map $W \rightarrow F \rightarrow M$ sends two elements of $W_{n}$ to the same element of $M$ if they describe equivalent braids (A0), (A1), (A2). A braid gives rise to a link by joining its top and bottom. By the classic theorem stated by Markov $[\mathbf{1 2}]$ and proved by Birman [3], two braids are sent to the same element in $M$ if they induce the same link (M1), (M2). The resulting function from links to $M$ satisfies condition one of the Main Theorem (P1). Invert the isomorphism to obtain an $R$-valued function that satisfies both conditions.

To prove the lemma consider the following six directed substitution rules (still with $u, v \in W_{n}, 0<b<a<n$ ):

$$
\begin{aligned}
& {[u a b v]_{n} \rightarrow[u b a v]_{n} \quad \text { for } b<a-1} \\
& {[u a(a-1) \cdots(b+1) b a v]_{n} \rightarrow[u(a-1) a(a-1) \cdots(b+1) b v]_{n}} \\
& {[u(-a) v]_{n} \rightarrow-x y^{-1}[u a v]_{n}-y^{-1} z[u v]_{n}} \\
& {[u a a v]_{n} \rightarrow-x^{-1} y[u v]_{n}-x^{-1} z[u a v]_{n}} \\
& {[u n v]_{n+1} \rightarrow[u v]_{n}} \\
& {[u v]_{n+1} \rightarrow-(x+y) z^{-1}[u v]_{n}}
\end{aligned}
$$

LEMMA. These rules obey the DCC: that is, there is no infinite sequence of correct applications starting with a single form.

LEMMA. Suppose that $f$ is a form and that it is possible to apply a rule to obtain the form $g$ and to make another application (of possibly another rule) to obtain the form $h$. Then there is a form $j$, a sequence of applications from $g$ to $j$, and a sequence of applications from $h$ to $j$. 
An inductive proof then shows that starting with any form $f$ and arbitrarily applying the rules, one inevitably arrives at a unique "terminal form" $T(f)$ on which no rule applies.

\section{LEMMA. A form is terminal iff it is an $R$-multiple of [ $]_{1}$.}

The Main Theorem is thus established with

LEMMA. $T(f)=T(g)$ iff $f \equiv g$.

For the most part these four lemmas have straightforward (but tedious) proofs. The DCC lemma needs a "complexity" measurement on forms guaranteed to decrease under each of the substitution rules. The next lemma reduces to 21 separate cases, one for each pair of rules. Each is proven by the simple expedient of applying in sequence the unique rule that applies. The characterization of terminal forms is achieved by finding at least one rule that applies to $[u]_{n}$ for $n>1$. The last lemma is easy if (M1) is deleted from the relations. ((M1) is in fact a consequence of the other relations.) The proof that $T[u v]_{k}=T[v u]_{k}$ quickly reduces to the case $T[n u v]_{n+1}=T[u v n]_{n+1}$, where $u$ and $v$ are descending words of positive elements, $u$ starting with $n-1$, $v$ with $n$. If $v$ is empty or a singleton or if $u$ is empty, a mindless verification works. Separate arguments are needed for the two remaining cases ( $u$ of length one, $u$ of length greater than one). If long computational proofs are acceptable, a mindless approach works for both (as it must if these lemmas are true): Apply any sequence of applicable substitution rules.

\section{The Hoste APPROACH}

We define $P$ as follows. Given any oriented link $L$ first choose some projection $S$ of $L$ and then "resolve" $S$ by first "changing" and "smoothing" some crossing in $S$, and then again changing and smoothing a crossing in each of the two projections that result from $S$, and so on until only unlinks remain. Next use property (1) of the Main Theorem and the values of $P$ for the unlinks given in Remark (1) to obtain a value of $P$ for $S$. We prove that the polynomial so produced is well defined and depends only on the isotopy class of $L$.

To avoid difficulties that arise from the fact that $S$ can be resolved in infinitely many ways, we begin with a slightly different definition of $P$. Given $S$, first order the components of $S$ and also distinguish a point on each component. We may then gain more control on the resolution of $S$ by first giving a rule whereby an ordering and pointing is induced on projections obtained from $S$, and then demanding that the resolution ends, not just in unlinks, but in "descending" projections. (A pointed ordered projection is descending if one never crosses over one's path while traversing the components in the given order and direction starting on each at the distinguished point.) Now use this "distinguished" resolution to compute $P$ of $S$, denoted $P_{S}$.

We next show $P_{S}$ is well defined-i.e., independent of the choice of ordering, pointing, and distinguished resolution, by induction on the number of crossings in $S$. To prove the inductive step we first show the choice of resolution is immaterial. This is made possible by the pointing and ordering 
scheme, which essentially implies that any two resolutions must eventually change all the same crossings, but perhaps in different orders. Employing the inductive hypothesis, it remains only to prove that (1) changing a crossing and immediately changing it back has no effect, and (2) changing the $i$ th and then the $j$ th crossings has the same effect as first changing the $j$ th and then the $i$ th crossings. Next we show that $P_{S}$ is unchanged if $S$ is repointed. This can be reduced to the case where $S$ is descending and one distinguished point is moved forward along its component past one crossing. Finally, we prove $P_{S}$ is independent of the ordering of $S$. This is the hardest step, although we can first reduce to the case where $S$ has two components and is descending, but not with respect to its own ordering. Now we show that $S$ can be transformed into a split descending projection (one where the components do not cross each other and, hence, the ordering does not matter) by a finite sequence of Reidemeister moves that never increase the number of crossings (so we can make use of the inductive hypothesis) and, furthermore, preserve $P_{S}$.

Once $P_{S}$ is known to be well defined, it is not hard to show that it is preserved by Reidemeister moves. Thus it depends only on the isotopy class of $L$. At this point we finally know that any distinguished resolution of the unlink produces the unlink polynomial, and from this we can prove that any resolution can be used to compute $P$, not just ones that are distinguished with respect to some choice of pointing and ordering of $S$.

\section{THE LICKORISH AND MILletT APPROACH}

Once the announced Main Theorem is believed, the only difficulty is proving that $P_{L}$ can be well defined; uniqueness, calculations, and Remark (4) are easy. Our combinatorial proof has simplicity: Let $\mathcal{L}_{n}$ be all planar projections of oriented links of at most $n$ crossings with components ordered and base-pointed. If $L \in \mathcal{L}_{n}$ let $\alpha L \in \mathcal{L}_{n}$ be $L$-with-changed-crossings so that $\alpha L$ ascends from the base point of the first component onwards (thus, following along the components of $\alpha L$ in the given order, always beginning at base points, a crossing is always first encountered as an underpass). Initially $P$ is defined as a function $P: \bigcup_{n} \mathcal{L}_{n} \rightarrow \mathbf{Z}\left[l^{ \pm 1}, m^{ \pm 1}\right]$. For $L \in \bigcup_{n} \mathcal{L}_{n}$ define $P_{\alpha L}=\mu^{c L-1}$, where $\mu=-\left(l+l^{-1}\right) m^{-1}$, and $L$ has $c L$ components. Inductively, assume that on $\mathcal{L}_{n-1}, \mathcal{P}$ has been defined, is independent of base points and orders, and

$$
l P_{L_{+}}+l^{-1} P_{L_{-}}+m P_{L_{0}}=0 .
$$

For $L$ in $\mathcal{L}_{n}$ define $\mathcal{P}_{L}$ to be the polynomial obtained from $\mathcal{P}_{\alpha L}$ using $(*)$ (and the inductive definition) on a sequence of crossing changes that creates $\alpha L$ from $L$. The choice of sequence is irrelevant, $(*)$ holds in $\mathcal{L}_{n}$, and $\mathcal{P}_{L}$ is unchanged by those Reidemeister moves that remain within $\mathcal{L}_{n}$. If now $\beta L$ denotes the new ascending element of $\mathcal{L}_{n}$ formed from $L$ by reference to a new set of base points and component order, the induction step is completed by showing that $P_{\beta L}$, as calculated from $P_{\alpha L}$, is $\mu^{c L-1}$. A straightforward argument on sliding base points along components shows (using the prescribed value of $\mu$ ) that this is so if only base points are changed; a change of component order requires a very careful use of the permitted Reidemeister moves on 
$\beta L$ to change $\beta L$ to another ascending projection with fewer crossings, thus determining $P_{\beta L}$ by induction. This done, $P_{L}$ is defined independent of base point and ordering; it is invariant under all Reidemeister moves, and the Main Theorem is proved.

The entire dependence of $\mathcal{P}_{L}$ on $(*)$ demands a redevelopment of Conway's skein theory $[\boldsymbol{7}]$ in the two-variable context. At once this new version of linear skein theory produces the elementary results that $P_{L_{1} \# L_{2}}=P_{L_{1}} P_{L_{2}}=$ $\mu^{-1} P_{L_{1} \sqcup L_{2}}$. Applied to two-string tangles $[\mathbf{1 1}]$, it shows that $P_{L}$ is unchanged by mutation (rotation through $\pi$ of such a tangle) in $L$. Thus the two elevencrossing knots (of distinct genera) for which $\Delta(t)=1$ have the same $P(l, m)$, which is, by calculation, not trivial (so $P_{L}$ depends on more than the Alexander module of $L)$. Also, mutation shows that for a pretzel knot $K\left(a_{1}, \ldots, a_{r}\right), \mathcal{P}_{K}$ is unchanged by permutation of the $a_{\imath}$. Further, if $S$ and $T$ are two-string tangles, if $S+T$ is

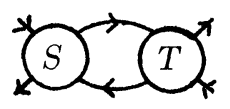

and $S^{N}$ and $S^{D}$ are the polynomials for

then

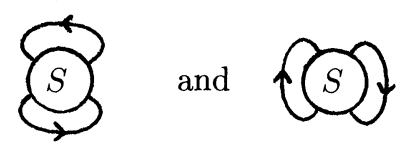

$$
\left(1-\mu^{2}\right)(S+T)^{N}=\left(S^{N} T^{D}+S^{D} T^{N}\right)-\mu\left(S^{N} T^{N}+S^{D} T^{D}\right) .
$$

This can be much applied in producing formulae for the polynomials of rational and other arborescent links. Two knots of Birman [4] with distinct signatures have the same polynomial. Thus if signature is a skein invariant, then $P$ is not injective on the set of skein-equivalence classes of oriented links. Two-variable analogues exist for most of the other skein-theoretic results given in $[7]$.

Tables will be produced (with computer aid) of the values of $\mathcal{P}$ for oriented links of low crossing number. At present the literature lacks an agreed notation and even a set of diagrams for oriented links. Manual calculations of the polynomials proceed via various neat recursion formulae for particular types (e.g., rational and pretzel) of links. Calculations of M. B. Thistlethwaite show that if $K$ is the knot $11_{388}$ (in the notation of K. Perko) and $\bar{K}$ is its mirror image, then $\mathcal{P}_{K} \neq \mathcal{P}_{\bar{K}}$, but both $V_{K}=V_{\bar{K}}$ and $\Delta_{K}=\Delta_{\bar{K}}$.

\section{The OCNEANU APPROACH}

We define for each permutation in $S_{n}$ a layered braid in the $n$-string braid group $B_{n}$. We show combinatorially that under the equivalence relation in the Main Theorem, any braid decomposes uniquely as a linear combination of layered braids. With the coefficients of the decomposition we construct invariants to conjugation in $B_{n}$, and obtain the invariant $P_{L}$ of the closed braid as an invariant to the Markov moves $[\mathbf{3}]$.

We then study the algebraic structure of the invariant $P_{L}$. Linear combinations of braids in $B_{n}$ have an algebra structure in which layered braids form 
a basis. The algebra turns out to be the Hecke algebra $H_{n}$ with Dynkin diagram $A_{n}[\mathbf{5}]$. In this way we explicitly construct a family of finite-dimensional irreducible representations of $B_{n}$, indexed by $n$-Young diagrams. The invariant appears as a weighted trace, explicitly determined via the $S$-functions associated to the discrete Euler $\beta$-function [13].

We then study the values of the variables for which the representation has additional orthogonality and positivity structure.

THEOREM. Let $\tau \in \mathbf{C}$. The free $C^{*}$-algebra $A_{\tau}$ over $\mathbf{C}$ generated by $\left\{e_{k} \mid k=1,2, \ldots\right\}$, satisfying $e_{k}^{2}=e_{k}^{*}=e_{k}$ and

$$
\begin{aligned}
e_{k} e_{k+1} e_{k}-\tau e_{k} & =e_{k+1} e_{k} e_{k+1}-\tau e_{k+1}, \quad k \in N, \\
e_{k} e_{l} & =e_{l} e_{k} \quad \text { if }|k-l| \geq 2,
\end{aligned}
$$

is nonzero if and only if $\tau \in\left[0, \frac{1}{4}\right] \cup\left\{(4 \cos (\pi / n))^{-1}, n=3,4,5, \ldots\right\}$.

TheOREM. Let $\alpha, \theta \in \mathbf{C}$, and let

$$
\tau=\left(4 \cosh ^{2} \theta\right)^{-1}, \quad \eta=\frac{1}{2}(1-\tanh \alpha \tan \theta) .
$$

Consider on the $C^{*}$-algebra $A_{\tau}$ a positive linear functional $\mu=\mu_{\tau, \eta}$ satisfying $\mu(x y)=\mu(y x)$, i.e., $\mu$ is a trace, $x, y \in A_{\tau}$, and $\mu\left(w e_{n+1}\right)=\eta \mu(w), w \in A_{\tau, n}$. Such a functional $\mu$ exists if and only if $\tau, \eta$ are given by one of the following values of $\alpha, \theta$ :

(i) $\theta \in \mathbf{R}^{+}, \alpha \in \mathbf{R}$ (the normal range);

(ii) $\theta \in \mathbf{R}^{+}, \alpha=k \theta+\pi i / 2, k \in \mathbf{Z} \backslash\{0\}$ (the exceptional lines);

(iii) $\theta=\pi i / m, m=3,4,5, \ldots ; \alpha=(k / m+1 / 2) \pi i, k=1,2, \ldots,[(m-1) / 2]$

(the exceptional points),

or, if $0 \leq \eta \leq 1$ and $\tau=\eta(1-\eta)$ (the limit line).

The trace $\mu$ is then uniquely determined and the corresponding weak closure $R_{\tau, \eta}$ of $A_{\tau}$ is the hyperfinite $\mathrm{II}_{1}$ factor for $\eta \neq 0,1$, and $\mathbf{C}$ for $\eta=0,1$.

For $\alpha, \theta$ as above consider the representation $\gamma=\gamma_{\alpha, \theta, \eta}$ of the braid group $B_{n}$ into $R_{\tau, \eta}$ given by

$$
\gamma\left(\sigma_{k}\right)=i\left(e^{-\alpha-\theta} e_{k}-e^{-\alpha+\theta}\left(1-e_{k}\right)\right), \quad k=1, \ldots, n-1 .
$$

Consider on $R_{\tau, \eta}$ the trace

$$
\phi=\phi_{\alpha, \theta}=(i \cosh \alpha \operatorname{csch} \theta)^{n-1} \mu_{\tau, \eta} .
$$

THEOREM. For $b \in B_{n}$ the invariant polynomial $P_{\hat{b}}$ of the closure $\hat{b}$ is given by $P_{\hat{b}}\left(e^{\alpha},-e^{-\alpha}, 2 i \sinh \theta\right)=\phi(\gamma(b))$.

$P_{L}$ is given by a positive trace of a unitary representation precisely at the exceptional points. The Jones invariant corresponds to the line $k=2$ and the Alexander invariant to $k=0$; the Arf invariant to $m=4, k=2$. For $k=1$, $P_{L} \equiv 1$, and for $k=-1, P_{L}= \pm 1$. This shows that for any knot $K, P_{K}-1$ is divided by $P_{\text {trefoil }}-1$. 
We have implemented a program which computes the coefficients of $P_{L}$.

REMARK. H. Wenzl has independently obtained the structure of the simple modules of the Hecke algebras by studying subfactors of the hyperfinite $\mathrm{II}_{1}$ factor and has computed the index and entropy of the subfactor generated by $e_{2}, e_{3}, \ldots$ in $R_{\alpha, \theta}$.

\section{REFERENCES}

1. E. Artin, Theorie der Zopfe, Hamburg Abh 9, pp. 47-72.

2. J. W. Alexander, Topologncal invariants of knots and links, Trans. Amer. Math. Soc. 30 (1928), 275-306.

3. J. Birman, Braids, links and mapping class groups, Ann. Math. Studies 82 (1974).

4. - On the Jones polymomial of closed 3-braids (to appear).

5. N. Bourbaki, Groupes et algèbres de Lie, Chaps. 4, 5, 6, no. 1337, Hermann, Paris, 1960-1972.

6. J. H. Conway, An enumeration of knots and links, Computational Problems in Abstract Algebra, Pergamon, N.Y., 1970, pp. 329-358.

7. C. Giller, A family of links and the Conway calculus, Trans. Amer. Math. Soc. 270 (1982), $75-109$.

8. V. F. R. Jones, Index for subfactors, Invent. Math. 72 (1983), 1-25.

9. __ Brand groups, Hecke algebras and type $\mathrm{II}_{1}$ factors, Proc. Japan-U.S. Conf., 1983 (to appear).

10. , A polymomzal invarant for knots via von Neumann algebras, Bull. Amer. Math. Soc. 12 (1985), 103-112.

11. W. B. R. Lickorish, Prme knots and tangles, Trans. Amer. Math. Soc. 267 (1981), $321-332$.

12. A. A. Markov, Über die freve Aequivalenz geschlossener Zopfe, Mat. Sb. 1 (1935), 73-78.

13. A. Wasserman, Thesis, Univ. of Pennsylvania, 1981.

Department of Mathematics, University of Pennsylvania, PhiladelPhia, PEnNSylvania 19146 (Current address of P. Freyd)

Department of Mathematics, Clark University, Worcester, MassaCHUSETTS 01610 (Current address of D. Yetter)

Department of MAthematics, Rutgers University, NeW BRUnswick, NEW JERSEY 08903 (Current address of J. Hoste)

Department of MAthematics, University of CAMBridge, CAMbridge, ENGLAND (Current address of W. B. R. Lickorish)

DEPARTMENT OF MATHEMATICS, UNIVERSity OF CALIFORNiA AT SANTA BARBARA, SANTA BARBARA, CALIFORNiA 93106 (Current address of K. Millett) AND MAthematical SCIEnCEs ResearCh Institute, Berkeley, California 94720

Mathematical Sciences Research institute, Berkeley, California 94720 (Current address of A. Ocneanu) 